scheinlich ebenfalls durch diese selbständige Thätigkeit der Membrana propria, der Abschluss gegen den Ausführungsgang.

Ich begnüge mich mit der blossen Angabe dieser Theorie; die Untersuchung über ibre Richtigkeit liegt ausserbalb des Zwecks meiner Arbeit. Nur kurz möchte jch noch die practische Schlussfolgerung anführen, die aus den Resultaten der Untersuchung beider Geschwülste zu ziehen ist, und ich glaube, sie liegt uns nicht sehr fern: da benigue, als solche längere Zeil bestehende Mammatumoren die Fähigkeit in sich tragen, einen malignen Charakter anzunehmen, so ist ihre Exstirpation möglichst bald vorzunehmen.

\title{
XXVI.
}

\section{Experimentelle Untersuchungen über die Resorption und Wirkung des regulinischen Quecksilbers der grauen Salbe.}

Von Dr. Paul Fürbringer, Prof. e. o. an der Lniversität Jena.

Seit einem Vierteljahrhundert hat es sich eine nambafte Reihe von Forschern angelegen sein lassen, auf dem Wege des Experjmentes die Frage nach der physiologischen Wirkung des Unguentum cinereum zu beantworten. Wie wenig mit den Resultaten für einen definitiven Abschluss dieser Frage gewonnen ist, dafür dürfte der in der soeben erschienenen Auflage des Nothnagel-Rossbach'schen Handbuches der Arzneimittellehre fixirte Status praesens besonders bezeichnend sein: Rossbach glaubt, dass bei der gangbaren Inunctionsweise der genannten Salbe das Quecksilber weniger durch die Haut als vielmehr durch die Athmungsorgane in Dampfform aufgenommen wird, hält es übrigens auch für möglich, dass der Metalldampf auch durch die unverletzte Haut, so wie die Metallkügelchen durch das lädirte (in Folge der Einreibung stellenweise der Epidermis beraubte) Integument in den Körper gelangt. "Dieser feine Metalldampf mag dann in dem Blute und den Organen unter dem Einfluss des Kochsalzes, des Blut- und Organeiweisses, des Sauerstoffs der Blutkörperchen zum Theil oxydirt, zum Theil 
vielleicht auch unverändert durch die Gewebe hindurchwandern und als metallisches Quecksilber wieder in den Se- und Excreten erscheinen."

So lautet beutzutage die Lehre von der physiologischen Wirkung des Unguentum cinereum. Dass sie einen so wenig affirmativen Ausdruck gefunden, muss seinen besondern Grund haben. Er erhellt bei Durchsicht der einschlägigen Literatur, welche zwei trotz ihrer vielfachen Ventilation noch als offen $z \mathrm{u}$ betrachtende Cardinalfragen aufweist:

1) Gelangt das metallische Quecksilber der grauen Salbe bei ihrer gewöhnlichen Applicationsweise als solches in das Innere des Körpers?

2) Wird das Metall durch Contaet mit der Säftemasse des Körpers oxydirt resp. in die wirksame lösliche Form umgewandelt?

Auf No. 1 haben die Versuchansteller in nahezu regelmåssiger Abwechselung bald Ja, bald Nein geantwortet; No. 2 hat fast nur im Reagenzglas eine Bearbeitung erfabren, deren Resultate auf den lebenden Organismus übertragen worden sind.

Beide Fragen habe ich aufgenommen und durch während Jahresfrist an Thier und Mensch angestellte Experimente zu erledigen gesucht.

I. Gelangt bei der Inunctionskur das metallische Quecksilber der grauen Salbe als solches in die Säftemasse des Körpers und auf welchem Wege?

Wir sehen hier einstweilen ab von den Schicksalen älterer d. i. aucb durch ibren Gehalt an fettsaurem Oxydul wirksamen grauen Salben. Zur Zeit zweifelt Niemand mehr daran, dass ganz frisch bereitetes Unguentum cinereum ein einfaches Gemenge von regulinischem Quecksilber und Fett ohne Beimischung einer 0xydationsstufe des Metalls ist und dass ein solches Präparat eminenter Resorptionswirkungen fähig ist.

Auf dreifachem Wege ist eine Aufnahme des Metalls in das Innere des Körpers denkbar:

1) am Orte der Application durch mechanische Locomotion der Metallkügelchen in die Haut; 
2) durch Einathmung des Dampfes; Körpers.

3) durch Eindringen des Dampfes in das Integument des

1) Wie verhalten sich die Quecksilberkügelchen in der Haut am Orte der Inunction?

Es liegt mir fern, der Mittheilung der von mir gewonnenen Resultate für diese Frage einen erschöpfenden Literaturbericht vorauszuschicken. So ziemlich jede diesbezügliche Publication enthält ein mebr oder weniger vollständiges Depot der Aufzeichnung der früheren Versuche und Resultate. Nur Folgendes glaube ich aus dem reichen Vorrath zur Orientirung hervorheben zu sollen:

Nachdem C. Hofmann ${ }^{1}$ ) zu dem Schlusse gekommen, dass das metallische Quecksilber bei Inunctionen nicht in Substanz in den Kreislauf gelangt, und deshalb entgegen der Ansicht 0 esterlen's und Eberhardt's von der Wanderung der Kügelchen durch die Haut Bärensprung in der Annahme, dass die graue Salbe nur durch ihren Gehalt an Quecksilberoxydul wirkt, beigestimmt, theilt uns Voit in seiner bekannten Schrift "uber die Aufnahme des Quecksilbers und seiner Verbindungen in den Körper ${ }^{22}$ ) folgenden Befund an Schnitten inungirter Leichenhaut mit: Die ganze Epidermis von kleinen schwarzen z. Th. schwach metallisch glänzenden Körnchen durchsät, einzelne derselben, mit einer „0xydationsschicht" überzogen, im Corium. Overbeck ${ }^{3}$ ) trifft die äussersten Vorsichtsmaassregeln, dass nicht etwa von aussen durch Hand oder Instrumente Quecksilberkügelchen in die Untersuchungsobjecte oder aber bei den Thieren selbst durch Ablecken etc. in den Mund und Magen gelangt sein konnten, und findet trotzdem bei seinen zahlreichen Einreibungsversuchen auf das Bestimmteste die (im Wesentlichen nicht oxydirten) Metallkügelchen in der Tiefe der eingeriebenen Hautpartien, im Blut, in den Organen, ja selbst im Harn und Koth wieder.

Ueber die Aufnahme der Metallkügelchen Seitens der Drüsengänge der Haut meldet uns Zuelzer in einer auffallenderweise

1) Ueber die Aufnahme des Quecksilbers und der Fette in den Kreislauf. Dlss. jnaug. Würzburg 1854.

2) Physiolog.-chemische Untersuchungen. 1. Heft. S. 45. Augsburg 1857.

s) Mercur u. Syphilis. Berlin 1861. S. $17 \mathrm{ff}$. 
vielfach übersehenen Abhandlung ${ }^{1}$ ) positive Resultate. Es gelang $\mathrm{ibm}$, in der mit grauer Salbe eingeriebenen und durch ein Blasenpflaster abgehobenen Epidermis die meisten der abgerissenen Ausführungsgänge, sowie die Haarwurzelscheiden mit Metallkügelchen gefüllt $\mathrm{zu}$ entdecken.

Nachdem nocb einmal Blomberg der Wanderung der Metallkügelchen durch die Haut das Wort geredet, begegnen wir der Behauptung Rindfleisch's ${ }^{2}$, dass auch nicht ein sichtbares Kügelchen weder die Epidermis durchdringt, noch in die Haarsäcke und Talgdrüsen eindringt. Dieser Ausspruch basirt auf folgender Versuchsanordnung: Das inungirte und gewaschene Kaninchenohr wird vom Körper getrennt, ein Stückchen Haut umschnitten und obne Anwendung eines Instrumentes mit der äusseren Seite auf den Objectträger gebettet. Die Wiederholung des Versuchs an der Haut von Leichen führte zu demselben Resultate.

Mit diesen Untersuchungen schien die Frage erledigt, bis J. Neumann ${ }^{3}$ ) mit dem Resultate zahlreicher, noch lange nicht genug gewürdigter Experimente an lebenden Thieren und Menschen zwar die Behauptung Rindfleisch's in Betreff des Coriums und Unterhautbindegewebes bestätigt, indess das Eindringen der Kügelchen in die Haarfollikel längs der Haare, sowie in die Talgdrüsen constatirt und diesen Befund durch beigefügte Abbildung in der überzeugendsten Weise illustrirt.

Die Reihe der Experimente schliesst ab mit den Versuchen von Auspitz ${ }^{4}$ ) und R. Fleischer ${ }^{5}$ ). Ersterer negirt ein reichliches Vorkommen von sichtbaren Metallkügelchen in der Lederhaut und dem Unterhautbindegewebe, letzterer vermochte nie Metallkügelchen in den Haarfollikeln, in den Talg- und Schweissdrüsen, ebenso wenig im Rete Malpighii und im Corium zu entdecken, schliesst

t) Ueber die Absorption durch die äussere Haut. Wien. Medicinal-Halle. 1864.

2) Zur Frage von der Resorption des regulinischen Quecksilbers. Archiv f. Dermat. u. Syph. 1870. S. 309.

3) Ueber die Aufnahme des Quecksilbers durch die unverletzte Haut. Wien. med. Wochenschr. 1871. No. 50-52.

4) Ueber die Resorption ungelöster Stoffe bei Säugethieren. Wien. med. Jahrbücher. 1871. S. 316.

5) Untersuchungen über das Resorptionsvermögen der menschlichen Haut. Errangen 1877. S. 75 . 
sich also den Rindfleisch'schen Beobachtungen an, nachdem diese bereits von Röhrig ') bestätigt worden waren.

Es müssen verschiedene Fehlerquellen concurrirt haben, dass so direct sich widersprechende Resultate gefördert werden konnten. Verwechslung von. Luftbläschen, Fetttröpfchen, Pigmentpartikeln mit Quecksilberkügelchen, Verunreinigung der Präparate mit Spuren grauer Salbe, Ablecken der eingeriebenen Hautbezirke Seitens der Thiere nach Entfernung selbst der festesten Verbände hat man in dieser Beziehung mehr oder weniger urgirt. Eine grössere Bedeutung möchte ich indess zwei andern Fehlerquellen beimessen; einmal dem bei der gewöhnlichen Schnittührung nur schwer zu vermeidenden Hineinziehen von Quecksilberkügelchen aus der äussersten Hornschicht in das Rete Malpighii und die Lederhaut; je mebr man es nach und nach verstanden, diese Möglichkeit auszuschliessen (wie Rindfleisch durch directes Abpräpariren auf den Objectträger, Neumann durch Beseitigung der Epidermis mittelst siedenden Wassers etc.), desto übereinstimmender und bestimmter werden die negativen Befunde im Corium. Die zweite Fehlerquelle ist meines Erachtens in der Art der - mit der jetzt an unseren Kranken geübten Einreibungskur nicht entfernt vergleichbaren Inunction der zarten Thierhaut gegeben. Wenn, wie uns Overbeck berichtet, Kaninchen und Hunde wochenlang mit grossen Mengen grauer Salbe täglich, womöglich an derselben Stelle eingerieben werden, so dürfen wir wohl den Nachweis der Kügelchen in den inneren Organen mit schweren Läsionen der Haut und in der Mundhöhle ("Gangrän der Schleimhaut") in Zusammenhang bringen; es wäre wunderbar, wenn die aufgeriebenen Hautcapillaren und die Mundgeschwüre nicht zahlreiche kleinste Kügelchen (z. Th. aus dem Metalldampf condensirt) aufgenommen hätten. Dass 0 verbeck durch auf diese Weise in die Circulation gelangtes metallisches Quecksilber getäuscht worden ist, scheint mir plausibler, als die Annahme einer Verwechslung von Luftbläschen etc. mit den Metallkügelchen ${ }^{2}$ ). Allein wie soll man die diametral entgegenstehenden

1) Die Physiologie der Haut. Berlin 1876. S. 108.

2) Die Schwierigkeit, kleinste Quecksilberkügelchen za erkennen und von Luftbläschen (Fetttröpfchen kommen wegen ihres Verbaltens zu Aether, Pigmentkörnchen schon wegen ihrer Configuration hier kaum in Frage) zu unterscheiden, ist nach melnen Erfahrungen niclit so überaus gross, als man sie 
Befunde im Bereich der Epidermoidalorgane erklären, die von Autoritäten wie Rindfleisch, Neumann u. A. vertreten werden? Dieser directe Widerspruch ist es vorwiegend gewesen, welcher mich zur Wiederholung der Versuche veranlasst hat.

Die Anordnung derselben vermeidet, wie ich glaube, alle die genannten Fehlerquellen dadurch, dass

1) sofort nach der einmaligen Inunction und Reinigung der betreffenden Hautpartie mit Seife dieselbe vom Körper getrennt wurde,

2) der Schnitt an völlig gehärteten Präparaten gegen die Hornschicht zu geführt wurde,

3) jch nur Kügelchen mit unzweifelhaftem Metallcharakter als solche angesprochen und schliesslich gar nicht mehr in die Verlegenheit kam, fragliche Gebilde berücksichtigen zu müssen (s. d. vorhergeh. und folg. Anm.).

Da es mir ferner galt, an den Thieren die correcte Inunctionskur, welche doch in der Regel die Haut unverletzt lässt, nachzuahmen und nicht durch übermässiges Einreiben schwere Hautläsionen herbeizuführen, habe ich niemals kürzer oder länger als 10-15 Minuten eingerieben und proportionale Mengen des Präparats (im Durchschnitt 0,1 ) angewandt. Auf diese Weise gelang

hingestellt. Hat man erst seine Augen an einigen Hunderten von Präparaten geübt, dann reducirt sich die Zahl der fraglichen Gebilde sehr erbeblich. Zu der Ueberzeugung, dass bel einer Vergrösserung von 3-400 sämmtliche Metallkügelchen überhaupt erkennbar sind, gelangt man leicht bei der Durchmusterung von Salbenresten, welche bereits zu Einreibungen gedient, sowie von feinsten Emulsionen des Metalls in Gummilösungen. Ich vermag Rindfleisch in seiner Behauptung nur beizustimmen, dass die kleinsten Kügelchen noch lange nicht die Grenze der Sichtbarkeit erreichen. Die Differentialdiagnose der Kügelchen und Luftbläschen anlangend kommt man zunächst schon mit den bekannten Charakteren (Metallkügelchen: dunkles Centrum, heller excentrischer, dem Beobachter zugewendeter Fleck, Luftbläschen: lichtes Centrum mit dunkler Peripherie) recht weit; Gebilde, die prima vista als einfache schwarze Punkte imponiren, deuten bei richtiger Einstellang jene Charaktere noch an. Die Zahl der - allerdings vorhandenen - ganz isolirten, allerkleinsten, durchaus fraglichen Partikel ist nicht so sebr bedeutend. Hier lässt sich nach meinen Erfahrungen auch keine mikrochemische Reaction, weder starke Salpetersäure, noch der Versuch einer event. Ueberführung in rothes Jodid, zumal wenn die Punkte im Hantgewebe eingebettet sind, verwerthen. 
es, die Epidermis vor Continuitätstrennungen za bewahren. Ueber Versuche an der verletzten Haut wird weiter unten berichtet werden.

Als Applicationsstellen dienten bei Kaninchen (Albinos) vorzugsweise die Löffel, beim Menschen und zwar beim gesunden (Excisionen kleiner Hautstückchen) wie an Moribunden (nachträgliche Wegnahme der Haut an der Leiche) die verschiedensten Körpergegenden. Die excidirten Stücke wurden sofort in absoluten Alkohol eingebracht, die Schnitte mit essigsäurehaltigem Glycerin oder Kalilauge aufgehellt. Dass auf äusserste Reinhaltung der Instrumente etc. gehalten wurde, versteht sich von selbst.

Es liegt nicht in meiner Absicht, über die Experimente (über 20) einzeln zu berichten. Ich erhielt im Allgemeinen an der Kaninchenhaut folgenden gemeinsamen Befund: Nirgends Continuitätstrennungen der Epidermis durch ihre ganze Dicke. Hornschicht streckenweise frei von jeder Spur von Quecksilber; an anderen Stellen einzelne und gehäufte Quecksilberkügelchen, welche hie und da unter abgelöste Zellenfetzen gerathen waren. Die tieferen Schichten gleich dem Rete Malpighii frei. Der grösste Theil der Haarsäcke Quecksilberkügelchen führend, bald vereinzelte kleinste, eben noch erkennbare, bald Gruppen solcher, bald zahlreiche bis zur Grösse von 0,0045 Mm. Durchmesser, fast durchweg kreisrund, metallisch glänzend mit spiegelndem excentrischen Fleck. Sämmtliche Kügelchen fanden sich zwischen Haarwurzel und innerer Wurzelscheide, und waren in einigen Präparaten bis in die Gegend der Papille gedrungen. Gleich den Haarfollikeln enthielt ein grosser Theil von Talgdrüsen in ihren Ausführungsgängen die Metallkügelchen und zwar sowohl in den direct nach aussen mündenden, wie in den in die Haartasche führenden. Mehrmals gelang es, eine schwarze, durchweg von Quecksilberkügelchen hergestellte Säule im peripherischen Theil des Haarfollikels zu entdecken, welche sich gablig spaltend in die Talgdrüsenausführungsgänge und den tieferen Haarsackabschnill fortsetzte. Endlich babe ich auch auf das Unzweifelhafteste die Metallkügelchen hie und da im Drüsenkörper selbst aufgefunden. Wurde der Schnitt der äusseren Hautfläche parallel geführt, so gewahrte man die zierlichsten Anordnungen der Metallkügelchen dicht um die Haarquerschnitte, kurz so überzeugende Bilder, dass Jeder, 
der meine Präparate gesehen, sie sofort richtig deutete. Was den Befund im Bereich der Cutis und des Unterhautbindegewebes anlangt, so muss ich offen bekennen, dass ich Anfangs lange geschwankt habe, ob nicht einzelne kleinste Kügelchen bis in die Gewebsspalten durcbgedrungen wären, bis ich endlich bei Beobachtung besonderer Cautelen zahlreiche Präparate erhielt, im Bereich welcher die Lederhaut weder erkennbare Quecksilberkügelchen, noch ibberhaupt fragliche dunkle Partikel aufwies ${ }^{1}$ ).

Der Befund an der menschlichen Haut entsprach im Wesentlichen dem gescbilderten. Die Untersuchungsobjecte stammten hier vorwiegend von den der Inunctionskur unterworfenen Krankęn meiner Abtheilung. Sofort nach erfolgter Inunction wurden die betreffenden Körperregionen (Streck- und Beugefläcben der Extremitäten, Brust, Bauch, Mons veneris etc.) von den Resten der grauen Salbe gereinigt, kleine spindelförmige Stückchen excidirt (bei sofortiger Sutur schnelle glatte Heilung per primam) und auf die beschriebene Art der mikroskopischen Untersuchung unterworfen. Die Beurtheilung des Verhaltens der Metallkügelchen in den Epider-

5) Die ersten Serien von Schnitten täuschten trotz aller vermeintlichen Vorsicht eine Aufnahme des Metalles durch die tieferen Epidermisschichten und namentlich das Corium und Unterhautbindegewebe vor, indem fast in allen Präparaten vereinzelte Quecksilberkügelchen in ihrer charakteristischen Erscheinungsform in den genannten Hautpartien sowie dem Obrknorpel unmittelbar benachbart angetroffen wurden. Diese kügelchen erweckten um so mehr den Eindruck der Einbettung in verschiedene Schichten des Gewebes, als je nach dem Grade der Einstellung des Mikroskopes bald diese, bald jene deutlich wurden, resp. verschwanden. Allein höchst verdächtig war die durch die Ränder der Schnitte gegebene Prädilectionsstelle, sowie der Umstand, dass inmitten der kleinsten Kügelchen Gebilde bis zu $0,005 \mathrm{Mm}$. und darüber auftauchten, die unmöglich die Epidermis durchwandert haben und in die Interstitien des Corium gedrungen sein konnten. Und in der That ergab eine sorgfâltige Prüfung, dass sämmtliche Kügelchen nur von aussen in die Schnitte gelangt sein konnten, einmal bereits im Härtungsglase durch Ablösung von der freien Hornschichtfläche und dann durch Hineinziehen des Inhaltes der Haarfollikel in die benachbarten Partien mit dem Messer. Die Trugbilder schwanden mehr und melir, sobald ich aus den mit viel Alkohol durchschüttelten gehärteten Hautstücken centrale Partien excidirte, gegen die Aussenflăche zu die Schnitte führte (event. nach Spaltung der Ohrenpartien inmitten des Knorpels in 2 Lamellen) und die Verschiebung der Schnitte auf dem Messer thunlichst zu vermelden verstand. 
moidalgebilden stösst hier wegen der Spärlichkeit der Talgdrüsen und Haare gegenüber den Verbältnissen am Kaninchenohr und namentlich der Misslichkeit, eine grössere Zahl dünner, senkrechter ${ }^{3}$ ), Haartasche und Drüsengang in einer Ebene aufweisenden Schnitte zu erhalten, auf Schwierigkeiten. Ich habe mehr als tausend Schnitte Revue passiren lassen müssen, ehe ich mit dem Resultat abschliessen konnte. Dasselbe bestand in Folgendem: Im Bereich der meisten Haartaschen konnte die Gegenwart der - spärlichen isolirten bis zahllosen gruppirten - Quecksilberkügelehen auf das Unzweideutigste erkannt werden ${ }^{2}$ ). Immer lagerten sie dem Haare selbst dicht an, waren an stärkeren Haaren nur selten über die Mitte des Balges hinaus zu verfolgen und erreichten nirgends, auch nicht an den Wollhaaren, wie beim Kaninchen, die Gegend der Papille. Vereinzelte Kügelchen erreichten die Grösse von $0,015 \mathrm{Mm}$. mit Andeutung von ovoider oder Nierenform; ihre Bildung schien durch Confluenz mehrerer eingepresster Kügelchen im Haarbalge bedingt. Die Ausführungsgänge der Talgdrüsen, von denen Neumann in seinen Versuchen an der mensehlichen Haut nichts erwähnt, wiesen

1) Flächenschnitte erzeugen hier, besonders von schnell gehärteten und in Folge dessen stark geschrumpften Präparaten, leicht Trugbilder: Der Grund der tiefsten Einbuchtungen der Epidermis, der trotz sorgfältigster Reinigung mit Seife zahlreiche Quecksilberkügelchen enthält, kann, ringsum von Corium umgeben, als Haartaschenquerschnitt und hie und da wohl auch als solcher von Drüsenausführungsgängen imponiren (cf. Hi ndfleisch, a. a. 0.).

2) Warum Rindfleisch zu negativen Resultaten gelangt, darüber wage lch keine bestimmte Meinung; indess kann fch nicht umhin, zu bekennen, dass auch ich Anfangs ab und za auf Hautstückchen obne jeden Metalleinschluss beim Kaninchen wie Menschen gestossen bin. Hieran trug, wie ich mich nachträglich ïberzeugt, die Art der Inunction die Schuld. Eigenthümlicher Weise wuchsen mit der Menge der verwandten Salbe die negativen Befunde, wie ich umgekehrt bei Benutzung geringer Mengen bis zur "Trockenreibung" (und diese erfordert eine regelrechte Inunctionskur) des geschilderten Verbaltens der eingedrungenen Kügelehen sicher sein konnte. In der That wird die graue Salbe ganz besonders in den letzten Stadien der Einreibung, wenn die fettig-geschmeidige Empfindung unter dem Finger schwindet und die Frietionen auf Widerstand stossen, befähigt, in die Blindsäcke der Haut einzudringen. Damit steht im Einklang, dass bei der Exstinction des Metalls mit Fett, Gummilösungen etc. (s. n.) ein Ueberschuss der Vehikel die Feinheit der Vertheilung zu beeinträchtigen, wenn nicht ganz zu vereiteln pflegt; man kann sich von dem "Ausweichen" der Metallkugeln in der Reibschale unter diesen Bedingungen sofort überzeugen. 
die Kügelchen ebeufalls auf, allerdings bei Weitem nicht in dem hohen Procentsatz, wie im Bereich der Kaninchenhaut; jene Gänge, welche in den Haarsack etwa in der Mitte desselben oder nur wenig oberbalb dieser einmündeten, zeigten sjch frei von Kügelchen, mebrfach dagegen fand ich sie in den oberflächlicher gelegenen Gängen und konnte endilich fast darauf rechnen, sie in Menge in den kurzen und relativ weiten Drüsengängen im Berejch der Wollhaare anzutreffen. Hier gelang es auch, an einigen Präparaten die Theilung des eingedrungenen Stromes in die Fortsetzung des Haarbalges und in den Talgdrüsengang zu beobachten. Im Drüsenkörper babe ich ausnahmslos die Kügelchen vermisst. Was endlich die Schweissdrüsen anlangt, so kann ich mich dem Befunde Neumann's nur anschliessen, indem ich die Anwesenheit der Quecksilberkügelchen nur im Bereich der konischen Mündung der Gänge constatiren konnte. Immerhin ist aber auch dieser Befund von Belang, weil das eingedrungene Metall, das nicht durch Waschungen oder Bäder entfernt wird, als Depot dem Körper zu eigen gegeben wird, sobald die Einreibung vollzogen. Im Bereich der tiefen Epidermisschichten und des Coriums war der Befund ebenso negativ wie in der Kaninchenhaut. Ich lehne also mit Hoffmann, Bärensprung, Rindfleisch, Röhrig, Neumann und Fleischer gegen Oesterlen, Eberhardt, Voit, Overbeck und Blomberg ein Wandern der Quecksilberkügelchen durch die intacte Epidermis und Lederhaut auf das Bestimmteste ab, behaupte aber eben so entschieden mit Zuelzer und Neumann gegen Rindfleisch und Fleischer ein Eindringen derselben in die Haarsäcke und Talgdrüsenausführungsgänge und erachte mit meinen Nachweisen die Frage für erledigt.

Wie aber, wenn die Haut vor oder während der Inunction Verletzungen, wie Epithelabschürfungen, Rhagaden etc. darbietet, resp. durch die Friction erleidet? Ich sage ausdrücklich vor oder während der Einreibung, weil das sog. Eczema mercuriale als nach derselben entstehend und für gewöhnlich eine weitere Benutzung der betroffenen Hautpartie für die Inunction verbietend, doch kaum in Betracht kommen kann. Aber auch das ersterwähnte Vorkommniss gehört erfahrungsgemäss nicht zur Regel, besonders die durch eine regelrechte Einreibung direct hervorgerufenen Epidermisdefeete, Blutungen u. s. w. Immerhin aber hat dies thatsächlich beobachtete Ereigniss uns aufgefordert, das Verhalten der 
Metallkügelchen bei Application der grauen Salbe auf die verletzte Haut zu studiren. Die Experimente bestanden im Anlegen seichter, die Epidermis durchdringender Einschnitte, sowie umschriebener Beseitigung derselben durch Ablösen oder Abschaben von den verschiedensten Hautpartien (besonders der Innenfläche der Kaninchenlöffel) und nachträglicher Inunction. Wäbrend derselben pflegen sich die verletzten Stellen deutlicher mit Blut zu umzeichnen. Nach dem Abwaschen und Härten erhielt ich an senkrechten Schnitten folgenden Befund: Im Bereich der Verletzung zahllose kleinste Metallkügelchen in den Interstitien des Coriums, das in unmittelbarer Nachbarschaft der Läsion dadureh ein dunkelgesprenkeltes Aussehen darbot. Schnelle Abnahme der Zahl der Kügelchen nach unten und seitwärts. Nur selten einzelne derselben in die tiefen Cutisschichten und das Unterhautbindegewebe verfolgbar. Da, wo verletzte Capillaren inmitten des Einwanderungsbezirkes aufgefunden werden konnten, zeigte sich ihr Inhalt ebenfalls von kleinsten Metallkügelehen durchsetzt. Aber auch noch ausserhalb des genannten Bezirkes waren vereinzelte Kügelchen zwischen den Blutkörperchen sichtbar.

Nach diesem Befunde kann es in der That keinem Zweifel unterliegen, dass bei der Inunctionskur am Orte der Application metallisches Quecksilber in die Circulation aufgenommen werden kann, wenn die Haut stellenweise jhres Epidermisüberzuges beraubt ist und klaffende Gefässe zu Tage liegen. -

2) Aufnahmedes metallischen Quecksilbers in Dampfform durch die Respiration.

Die Flüchtigkeit des metallischen Quecksilbers bei gewöhnlicher Temperatur resp. unter dem Einflusse der Körperwärme sowie die Aufnahme des Dampfes durch die Respiration bis zur prägnantesten Wirkung ist eine längst bewiesene Thatsache [Schiff Triumpf, gewerblicher Mercurialismus ${ }^{1}$ ), Intoxicationen in der Umgebung von Menschen, welche der Schmierkur unterworfen werden]. Zudem ist die Abgabe von Metalldampf Seitens der grauen Salbe bei Körpertemperatur von Fleischer (a. a. 0. S. 74) durch Experimente

1) Cf. vor Allem Ku ssmaul, Untersuchungen über d. constitut. Mereurialismus (Wärzburg 1861), S. 89, 220 ff. und Naunyn, "Quecksilbervergiftung" (Handbuch der Intoxicationen; Band XV der r. Ziemssen'schen spec. Path. u. Ther. 1876) S. 301. 
direct zur Anschauung gebracht worden. Wie steht es aber mit dem Schicksal des Metalldampfes im Bereich der Mund- und Respiratínsschleimhaut? Beim Contact von Dämpfen mit einer feuchten Oberfläche erfolgt nothwendig die Condensation der ersteren an letzterer. Es muss also bei der Aufnahme von Quecksilberdampf mit der Respiration derselbe sich an den betheiligten Schleimhäuten in feinen Tröpfehen regulinisch niederschla$\operatorname{ge~}^{1}$ ). Trotzdem dieser Satz bereits vor 23 Jahren, freilich in einer fast allgemein übersehenen Abhandlung ${ }^{2}$ ) ausgesprochen worden, des Ferneren Hermann in seiner Toxikologie ${ }^{3}$ ) ihn wiederholt mit dem Zusatze: „Dass Quecksilberdämpfe als solche durch die Lungen in's Blut aufgenommen werden können, ist eine falsche physikalische Vorstellung ", wird immer noch die Existenz des Quecksilberdampfes im Blute behauptet. Nothwendig muss sich der durch die Respiration aufgenommene Metalldampf im Bereich der Mundhöble und Respirationsorgane event. auch im Magen und Darm (Verschlucken des Mundinhalts) in Form regulinischer Kügelchen an den Respirationsschleimbäuten resp. im Mund- und Mageninhalt wiederfinden. Wissen wir des Weiteren durch die Rindfleisch'schen Untersuchungen (a. a. 0. S. 312 ff.), dass die Quecksilberkügelehen in die unverletzte Schleimhaut nicht eindringen, so werden wir eine Aufnahme des zu Tröpfehen verdichteten Dampfes in das Blut höcbstens für die gewiss nicht zur Regel zählenden Fälle einräumen, in denen es sich um Läsionen der betreffenden Schleimhâute handelt.

Diese Erwägungen erledigen auch zum grössten Theil die (3.) Frage nach dem Verhalten des Metalldampfes zur unverletzten Haut. Röhrig ist der Einzige, der dem Penetriren

1) Man kann dies durch Aufhängen feuchter Haut über erwärmtem Quecksilber zur Anschauung bringen. Nach längerer Einwirkung entdeckt selbst das freie Auge spiegelnde Kügelchen inmitten des grauen Metallbeschlages. Goldblättchen, welche Tage lang auf dem fenchten Darmverschluss eines mit Quecksilber gefültten Gefässes gelagert, gaben beim Erhitzen keine Spur Quecksilbers ab (eigner Versuch).

2) G. Lewald, Untersuchung über den Uebergang von Arzneimitteln in die Milch. Habil.-Abhandl. Breslau 1857. Abschnitt: Quecksilber (S. 23). Ich finde diesen Autor nur von Binz in den neueren Auflagen seiner „Grundzüge" erwähnt.

3) Berlin 1874. S. 212, ausserd. S. 239, Anm. 4. 
der Quecksilberdämpfe durch die Epidermis das Wort redet, allerdings „böchstens in geringen Spuren“ (a. a. 0. S. 106, 108). Es hat dies Fleischer (a. a. 0. S. 74) für die todte Haut experimentell negirt ${ }^{1}$ ). Eine andere Frage, die nicht von Letzterem berübrt worden, ist es indess, ob nicht feinster Metalldampf durch die trockne Hornschicht streichen kann, um sich innerbalb der übrigen feuchten Haut regulinisch $\mathrm{zu}$ verdichten. Ich habe diese Frage an der lebenden Haut zu lösen gesucht: Die Volarfläche des durchaus trockenen Unterarmes eines meiner Patienten wurde längere Zeit den Dämpfen erwärmten Quecksilbers ausgesetzt. Der Effect war, dass sich die betroffene Hautpartie mit einem mattgrauen Beschlage bedeckte, inmitten dessen die Lupe kleine spiegelnde Metallkügelchen, zumeist in dem Grunde der Epidermisfältchen sitzend, aufwies. Jener graue Schmauch wurde unter dem Mikroskop als lediglich von Metallkügelchen hergestellt erkannt. Nach dem Abspülen derselben wurde ein kleines Stückchen Haut excidirt und auf die bereits mehrfach erwähnte Weise behandelt. Es ergab sich, dass wohl vereinzelte Kügelchen der Aussenfläche der Epidermis als Rest des Beschlages anbafteten, dass indess auch nicht ein einziges zwischen den geschlossenen Zellen der Hornschicht oder im Rete Malpighii entdeckt werden konnte. Sämmtliche Drüsenausführungsgänge und Haarsäcke desgleichen vollkommen frei. Man muss also wohl die Vorstellung aufgeben, dass das unverletzte Integument metallisches Quecksilber in das Innere des Körpers aufnehmen kann, abgesehen natürlich von dem Orte der Inunction, wo, wie wir gesehen haben, durch die Einreibung massenhaft Metallkügelchen in Haarsäcke und Talgdrüsengänge eingepresst werden.

Das Verbalten der mit dem abdampfenden Quecksilber in Contact gelangenden, namentlich feuchten (schwitzenden) Hautbezirke ist indess insofern von Belang, als bekanntlich bei der Inunctionskur viel geschwitzt wird und die deshalb auf der Haut erfolgende Condensation des Metalldampfes za einer Ablagerung und Haftung von Kügelchen fübren muss. Es kann also das Integument eine ähnliche Rolle wie die Respirationsschleimhaut übernehmen.

1) Cf. auch die von Overbeck (a. a. O. S. 26) citirte Ansicht Michaelis': „Die Annahme, dass das metallische Quecksilber der granen Salbe in Gasform in den Kreislauf gelangen kann, ist kaum denkbar, " 
Selbstverständlich gilt von der verletzten Haut hier dasselbe wie von den lädirten Schleimhäuten. Eine Einwanderung regulinischen Metalls in das klaffende Gewebe ist wahrscheinlich. -

Kann es nach dem Vorstehenden keinem Zweifel unterliegen, dass bei der gewöhnlichen Application der grauen Salbe die unverletzte Haut am Orte der Einreibung regulinisches Metall in ihre Haarsäcke und Drüsen aufnimmit, dass durch den Act der Respiration das abdampfende Metall sich an den Schleimhäuten condensirt und an der Oberfäche des Körpers sich ebenfalls in Metalltröpfchen niederschlägt, so wird es sich zunächst und vor Allem fragen, wie das auf diese Weise dem Organismus zu eigen gegebene regulinische Quecksilber zur Wirkung gelangt. Diese Frage fällt, da nach durchaus übereinstimmendem Urtheil das Metall niemals als solches, sondern nur in oxydirtem und löslichem Zustande Resorptionswirkungen zu entfalten vermag, zusammen mit der 2. Cardinalfrage :

II. Wird das regulinische Quecksilber durch seinen Contact mit der Säftemasse des Körpers oxydirt resp. in die wirksame lösliche Form übergeführt?

Es liegt uns fern, uns bier mit der Art und Weise der chemischen Umwandlung des regulinischen Quecksilbers innerhalb des Organismus zu beschäftigen; die Untersuchungen Voit's bilden auch heute noch die Grundlage der Lehre von der Ueberführung auch der unlöslichen Quecksilberpräparate Seitens des Körpers in die Endproducte Chlorquecksilber-Chlornatrium und Quecksilberoxydalbuminat (endliche im Blute kreisende Verbindung). Vielmehr gilt es uns lediglich, den vor Allem für die practische Seite in Betracht kommenden Nachweis zu erbringen, dass das metallische Quecksilber im Organismus Bedingungen vorfindet, unter welchen es durch Oxydation und Löslichwerdung arzneilich wirksam wird.

Auch für diese Frage verfügen wir über eine stattlicbe Literatur. Es genüge hier die Angabe, dass auf Grund zahlreicher Versuche im Laboratorium (Behandeln von fein vertheiltem Quecksilber mit Kochsalz-, Eiweisslösungen, Blut etc.) die übereinstimmende Ansicht ausgesprochen wird, dass jene Oxydation zur löslichen Verbindung unter dem Einfluss des Kochsalzes, der Eiweiss- 
verbindungen, des Blutes des Körpers („Ozonbildung") erfolgt (Mialhe, Schönbein, Voit, Overbeck, Maly, Zeissl u. A.). Hervorzuheben ist, dass nach den Untersuchungen von Overbeck (a. a. 0. S. 288) bei diesen Versuchen dem Schilteln ein specifischer Einfluss zukommt. Das Verhalten des Metalls zum Hautsecret anlangend wird vielfach eine Ueberführung in die lösliche Form durch die organischen Säuren vermuthet (Michaelis, Neumann, Röhrig u. s. w.); indess nur Lewald (a. a. 0. S. 23 ff.) bat durch directe Versuche im Laboratorium dieselbe erwiesen: Die Behandlung feinvertheilten metallischen Quecksilbers mit buttersaurem Ammoniak, einem Bestandtheil des Hautsecretes, lieferte, ohne dass Schütteln nöthig gewesen, lösliches Quecksilbersalz. Der Sublimatbildung beim Contact des Quecksilberdampfes mit den Scbleimhäuten redet Kirchgässer ${ }^{2}$ ) das Wort, und Hermann endlich hält es für unwahrscheinlich, dass das in den Säften enthaltene metallische Quecksilber sich schliesslich genau wie resorbirte Salze oder Albuminate verhalte, „man müsste denn mindestens eine nachträgliche Oxydation innerhalb des stoffwechsels annehmen. Es ist aber viel wahrscheinlicher, dass schon bei der Ablagerung auf den von Luft bestrichenen Scbleimhäuten eine Oxydation des äusserst fein vertheilten Metalles stattfindet und sehr leicht möglich, dass das metallisch eingedrungene Quecksilber mit der Wirkung überhaupt nichts zu thun hat." Man sieht, die Frage ist fast nur mit Schüttelversuchen im Reagenzglas oder mit Vermuthungen beantwortet worden. Dem entgegen babe ich zunächst im Jahre 1878 directe Experimente am lebenden Organismus angestellt ${ }^{2}$ ) zur Lösung der von Hermann offen gelassenen resp. als unwahrscheinlich hingestellten Frage: Findet bei Abschlıss der Atmosphäre, also bei Ausschluss der Oxydation des regulinischen Quecksilbers durch Bestreichung der Luft eine nachträgliche Oxydation des Metalles innerhalb des Stoffwechsels (unter dem Einfluss der vitalen Säfte) statt?

Durch Einführung einer Quecksilberemulsion ohne Spur einer oxydirten Verbindung in den von der Atmosphäre abgeschlossenen

1) Ueber die Wirkung der Quecksilberdämpfe, welche sich bei Inunctionen mit grauer Salbe entwickeln. Dieses Archiv Bd. XXXII. S. 145.

2) Zar localen und resorptiven Wirkungsweise des subcutan injicirten metallischen Quecksilbers. D. Arch. f. klin. Med. XXIV. S. 129. 
Unterhautraum habe ich eine positive Antwort erhalten insofern als in dem hämorrhagischen Eiter eines subcutanen Abscesses, sowie in einem Theil der Harne der Behandelten lösliches Quecksilber in deutlichen Spuren gefunden wurde. Hiermit war der Nachweis erbracht, dass wenigstens ein mit Blut untermischtes pathologisches Product, der Eiter, durch einfachen Contact mit metallischem Quecksilber ohne Betheiligung der Luft von letzterem eine lösliche 0xydationsstufe abzuspalten vermag. Dass auch das Blut dieses Vermögen besitzt, ist durch den Nachweis löslichen Quecksilbers im Harn höchst wahrscheinlich geworden, allein in dieser Beziehung der Einwurf nicht sicher von der Hand zu weisen, dass feinste Kügelchen in den secretorischen Apparat der Niere gelangt und unter dem Einfluss des sauren Harnes lösliches Salz abgegeben.

Es galt also, den directen Nachweis der Ueberführung des Metalles in eine lösliche Verbindung Seitens der normalen Gewebssäfte innerhalb des lebenden Organismus ohne Betheiligung der Atmosphäre zu liefern. Ich habe dies durch eine Reihe im verflossenen Winter und Frühling methodisch angestellter Thierexperimente, über deren Resultat ich bereits vorläufig berichtet ${ }^{1}$ ), unternommen. Als Paradigma der Gewebssäfte war aus guten Gründen nur das im geschlossenen Röhrensystem kreisende Blut zu wählen, das ich in directen Contact mit feinvertheiltem Quecksilber zu bringen und nach gewisser Zeit auf die Anwesenheit löslicher Oxydationsproducte zu prüfen trachtete. Es gelang endlich nach Ueberwindung anscheinend unbesiegbarer Hindernisse [Tod der Thiere durch zahllose Embolien ${ }^{2}$ ) und metastatische Abscesse in Lunge, Leber etc., Unmöglichkeit der Verarbeitung des geronnenen

1) Experimentelle Untersuchungen über die Wirkung des iebenden Blutes auf metall. Quecksilber. Sitzungsberichte der Jenaischen Gesellschaft f. Med. u. Naturwiss, vam 7. Mai 1880.

2) Bei dieser Gelegenbeit sei bemerkt, dass die meinerseits anooncirten Thierexperimente zur Frage nach dem Verbleib des in die Vene injicirten laufenden Quecksilbers sehr bald sich in ihren Resultaten nur den früberen namentlich durch die Virchow'schen und Cobn'schen Versuche bekannt gewordenen Thatsachen anzuschliessen vermocbt und deshalb abgebrochen wurden. Offenbar waren bel den "Vorversuchen" die Embolien einfach aus Anlass der Stagnation des injicirten Metalls (mechanische Senkung, Abseliluss durch Thromben etc.) rermisst worden. Vgl. $Z$ aha, Untersuch, über Thromb. (dies. Archiv LXII.). 
Blutes unter Ausschluss von Fehlerquellen] die Herstellung einer sō extrem feinen Vertheilung des Metalles in einer Mischung von Gummischleim und Glycerin ${ }^{1}$ ), dass die zu Injectionen in den Kreislauf verwandten Mengen nur spärlíche, die Blutkörperchen an Grösse übertreffende Metallkügelchen enthielten; bei Benutzung derartiger Präparate, die sich frei von jeder Beimischung löslichen Quecksilbers zur Zeit der Versuche erwiesen, konnten die Thiere vor lebensgefäbrlichen Embolien ${ }^{2}$ ), ja in einigen Fällen von nachweisbaren Infarcten iiberhaupt bewahrt und bis za einer Woche (vielleicht auch noch viel länger) am Leben erhalten werden. Es glückte ferner die Gewinnung eines klaren von Blutkörperchen und namentlich Fibrinausscheidungen freien Blutserums, das sicher keine Spur der injicirten

1) Diese Emulsion habe ich selbst allwöchentlich frisch bereitet durch längeres Verreiben von 5,0 chemisch reinen Quecksilbers mit geringsten Mengen reinster Mucil. gummi arab. (eín Ueberschuss vereitelt die feine Vertheilung des Metalles) unter successivem Zusatz von diesem Vehikel und reinen Glycerins bis zu einem Gehalt von 15,0 Mucil. und 5,0 Glyc. Man erhält auf diese Weise ein der von mir früher zu den subcutanen Injectionen (s. o.) verwandten und beschriebenen Emulsion ähnliches dickflüssiges, dunkelmausgraues Präparat, welches beim Stehenlassen die grösseren Kügelchen zu Boden fallen lässt. Nach 10-20 Minuten entbält die überstehende Flüssigkeit, wie man sich unter dem Mikroskop überzeugen kann, überraschend fein vertheiltes Metall, derart, dass bei Durchmusterung eines Tropfens nur wenige oder geradezu keine die Blutkörperchen an Grösse übertreffende Kügelchen entdeckt werden. Die Oberflächenvergrösserung ist eine ungleich bedeatendere, als jene in der grauen Salbe, und mag bis in's Tausendfache, wenn nicht darüber gehen. Die Verarmung der Flüssigkeit an Metall beim Sedimentiren ist eine relativ erhebliche, letztere indess wegen ihrer andurchsichtigen Beschaffenheit noch immer als sebr quecksilberreich $z u$ bezeichnen. Sie enthält für die ersten Wochen nach ihrer Bereitung keine Spur eines löslichen Salzes, wie man sich durch Aufschütteln eines Quantums mit viel Wasser, tagelangem Stebenlassen (noch nach 2 Stunden habe ich in der dünnen anscheinend völlig klaren Flüssigkeit kleinste Quecksilberkügelchen entdeckt) und electrolytischer Behandlung der abgehobenen und mit $\mathrm{HCl}$ und $\mathrm{KClO}_{3}$ beschickten Flüssigkeit überzeugen kann. Nach langem Stehen in mangelhaft verschlossenen Gefässen erfolgt eine allmähliche Bildung einer löslichen Verbindung, die ich als schleimsaures Oxydul anspreche.

2) Capilläre Embolien im Bereich der Lungen habe ich nicht selten aufgefunden, obne dass die Thiere überhaupt zu Lebzeiten gelitten zu haben schienen. In einem Falle fand ich die Lungen durchaus intact und im Bereich der Nieren und einzelner Muskelbezirke Embolien. Hier hatte offenbar der Lungenkreislauf als gröberes Filter die kleinen Emboli passiren lassen. 
Kügelchen enthielt, und somit auf die Gegenwart einer löslichen Quecksilberverbindung auf electrolytisehem Wege geprüft werden konnte.

Der specielle Plan, nach dem ich verfuhr, war folgender: Den aufgebundenen und narcotisirten Thieren (Kaninchen, 1 grosser Hund) wurde $1 \mathrm{Ccm}$. der Emulsion in die freigelegte Jugularis oder Femoralvene injicirt. Das Injectum wirà bej einiger Uebung und richtiger Anordnung (Canülen- und peripherische Ligatur, sehr langsame Injection) vollständig und fast ohne allen Blutverlust aufgenommen; nur im Bereich des Contacts der Gefässwand mit der Canüle pflegt sich ein von Metallkügelèhen infiltrirter Thrombus zu bilden. Nach gewisser Frist (s. u.) wurde das Blut direct aus den durchschnitfenen Halsgefässen aufgefangen ${ }^{1}$ ) (Menge kaum $30 \mathrm{Ccm}$. im Durchschnitt), sofort defibrinirt und mit dem 5-10fachen Volum einer $3 \frac{1}{2}$ procentigen Kochsalzlösung versetzt. Nach 24 stündigem Stehen hatten sich die Blutkörperchen als Schlanm zu Boden gesetzt und das klare, schwachröthliche oder fast farblose, verdünnte Serum konnte abgehoben und zur Zerstörung der organischen Substanz mit Salzsäure und chlorsaurem Kali behandelt werden. Man erhält auf diese Weise ein farbloses, durchaus klares Filtrat, das man direct der Prüfung auf Quecksilber auf electrolytischem Wege unterwerfen kann. Ich benutzte ausschliesslich das von mir modificirte Ludwig'sche Verfahren ${ }^{2}$ ).

Bevor indess von einem etwa positiven Nachweise auf die Wirkung des Organismus geschlossen werden konnte, galt es dem efwaigen Einwurf zu begegnen: kann nicht lösliches Quecksilber entstehen durch die Manipulationen des Defibrinirens und durch die Belassung des Blntkörperchenschlamms, der ja auch zahlreiche Metallkügelchen einschloss, mit dem kochsalzreichen Serum im mangelhaft verschlossenen Gefäss? Diese Vermuthung hat sich mir nach mehrfachen Versuchen durchaus nicht bestätigt: frisches Kaninchenblut zu 20-30 Gcm. mit je $1 \mathrm{Ccm}$. der erwähnten Metallemulsion

1) Ein erheblicher Einfluss dieser Injectionen auf die Form der Blutkörperchen war nicht zu constatíren. Auch der Harn zeigte sich bämoglobinfrei. Von einer wesentlichen Glycerinwirkung lonnte schon aus Anlass der geringen Mengen und ihrer directen Injection in's Blut nicht die Rede sein.

2) Berliner klin. Wochenschrift. 1878. No. 23. Meine ,Messingwolle “, von Vulpius als Kupferbänder mit Messingüberzug erkanst, geht jetzt unter dem Namen Lametta (Arch. f. Augenbeilk. 1880.). 
gemischt und in gleicher Weise behandelt lieferte ein Serum, das sich constant als quecksilberfrei erwies. Ich zähle, wenn ich von allen nicht vollständig gelungenen Experimenten absehe, im Ganzen 12 geglückte Versuche mit folgendem Resultat:

Fünf Mal erhielt ich deutlicbste Quecksilber-Jodid-Ringe in den Capillaren; in diesen Fällen hatte ich die Thiere 24 Stunden, 2, 3, 5 und endlich 6 Tage nach der Injection getödtet, vier Mal (Untersuchung des Blutes 12, 24, 44 und 48 Stunden nach der Injection) war der Befund ein negativer und in den übrigen Versuchen (Tödtung der Thiere nach 2, 4 und 7 Tagen) insofern ein fraglicher, als bei Durchleitung des Joddampfes nicht überzeugende Jodidbeschläge auftraten. Was den negativen Befunden zu Grunde gelegen (individuell verschiedene Oxydationskraft des Thierkörpers? Beschlagnahme der Quecksilberverbindung durch die Blutkörperchen oder das Fibrin?) wage ich nicht zu entscheiden. Jedenfalls erbellt aus den genannten 5 positiven Resultaten, dass das lebende Blut bereits nach 24 stündigem und spätestens 6 tägigem Contact mit dem regulinischen Quecksilber von diesem eine lösliche d. i. wirksame Oxydationsstufe abzuspalten vermag. Die nachgewiesene Menge der letzteren war stets sehr gering, was in Anbetracht der eminenten Oberflächenvergrösserung des Metalles und somit ungemein multiplicirten Angriffspunkte des Blutes auf dasselbe auffällig erscheint. Dem gegenüber ist hemerkenswerth, dass, so oft ich die Leber der Thiere auf Jösliches Quecksilber prüfte (Filtrat des wässrigen Extracts nach 24 stündigem Stehen mit $\mathrm{HCl}$ und $\mathrm{KClO}_{3}$ behandelt) letzteres in reichlicher Menge nachgewiesen werden konnte.

Nach dem Verbleib der Quecksilberkügelchen habe ich wiederholt gefahndet. Ich fand sie meist in den Fibringerinnseln, sowie im Blutkörperchenschlamm in reichlicher Menge vor, besonders bei wiederholtem Aufgiessen. und Decanthiren im Grund des Spitzglases. Die meisten schienen, abgesehen von der offenbar durch secundäre Confluenz im Glase entstandenen Grösse einzelner unverändert, ein Theil jedoch wies charakteristische Veränderungen auf, die vorwiegend an den kleinsten Kügelchen beobachtet wurden. Die ersten Phasen gaben sich durch Minderung des Metallglanzes kund, derart, dass der belle Mittelkreis und besonders die spiegelnde Stelle derselben (s. o.) trüb bis fast unkenntlich erschien. Weiter schwand 
jeder Metallglanz und die kreisrunde Contour, die einer mehr eckigen Platz machte. Endlich erschienen tiefschwarze zackige Gebilde. In einem kleinen Thrombus der Saphena des erwähnten Hundes, dem ich 5 Tage vor der Tödtung $3 \mathrm{Cem}$. der Emulsion injicirt batte, fand ich neben anscheinend unverănderten und auffallend grossen Kugeln, die sich nur secundär durch Confluenz gebildet haben kommten, eckige, schwarze Kügelchen, die richtige Auslänfer in Form schwärzlicher, aber an den Rändern durchscheinender Spiesse getrieben hatten. Derartige Krystallfragmente fanden sich auch isolirt vor. Essig- und Salzsäure brachte die durchscheinenden Ränder zum Schwund; immer aber restirte ein glänzender Kern, der bei Behandlung mit starker Salpetersäure einschmolz, also offenbar aus Quecksilbermetall bestand.

Die beschriebenen Veränderungen stimmen im Wesentlichen mit dem von Voit und 0 verbeck an den Kügelehen in alter grauer Salbe sowie von mir an jenen alter Gummiglycerinemulsionen beschriebenen ${ }^{1}$ ) überein, ebenso mit der Beschreibung, die Rindfleisch von den im Darminhalt der Thiere aufgefundenen Quecksilbertheilchen und $\mathrm{Zahn}$ vou den in die Blutcirculation eingefübrten Metallkugeln giebt. Nach dem einstimmigen Urtbeil aller Autoren sind sie der Ausdruck einer Oxydation des Quecksilbermetalls ("Oxydulkruste", krystallinische Umgestaltung durch Einwirkung des Oxydationsprozesses von aussen), und wir werden somit uns vorstellen müssen, dass das in den Kreislauf gelangte Metall nicht ohne Weiteres durch Sublimatabspaltung einschmilat, sondern letzterer die Phase der 0xydulbildung vorangeht. Mit dieser Erwågung ist zugleich das Verhältniss der Bärensprung'schen Oxydulsalbe resp. alter oxydulbaltiger grauer Salbe zum frischen, lediglich Metall enthaltenden Unguentum einereum gekennzeichnet. Erstere sind allerdings den letzteren um ein Stück Oxydationsarbeit voraus, aber es fragt sich, was schon overbeck andeutet, ob die Oxydulpartikelchen bei der Inunction leichter in die genannten Hautgebilde einwandern, als die regulinischen Kügelchen, ganz abgesehen von der Rolle der Metalldämpfe.

1) Cf. Nachtrag II der Abhandlung im XXIV. Bande d. d. Arch. f. klin. Medicin. Hier waren die meisten Kügelchen eckig, sternförmig, mit z. Th. langen ausstrablenden Stäbchen verseben; letztere auch isolirt vorhanden oder verschränkte Gitter darstellend mit rundlichen Knotenponkten (ursprünglich Metallkügelchen). Alles Producte des schleimsauren 0xydationsprozesses. 
Wissen wir somit durch die vorstehenden Untersuchungen, dass überall da, wo bei der Inunction der grauen Salbe eine Aufnahme von Metallkügelchen in's Blut durch Bautläsionen etc. stattfindet, die Folge der Contactwirkung in der Bildung einer löslichen wirksamen Oxydationsstufe des Metalles gegeben ist, so wird es sich des Weiteren fragen, was mil dem in den Haartaschen und Talgdrüsen, sowie a uf den Schleimhäuten und a uf der unversehrten Haut abgelagerten regulinischen Quecksilber geschieht.

Die Schicksale der in den Hautblindsäcken deponirten regulinischen Kügelchen anlangend, verfügen wir über eine werthvolle auffallenderweise kaum bekannt gewordene Beobachtung Neumann's. Derselbe constatirte (a. a. 0, S. 1236), dass jene Quecksilberkügelchen 4 Wochen nach der Inunction geschwunden waren, indem er zur angegebenen Zeit die Obrenstumpfe des eingeriebenen Kaninchens einer nochmaligen Untersuchung unterwarf. Ich habe diesen Schwund nicht abgewartet, sondern mich durch eine Nachuntersuchung bereits 8 Tage nach der Inunction (successives Abtragen der Löffel vom lebenden Thiere) von der Beschaffenheit der eingedrungenen Quecksilberkügelchen überzeugt. $\mathrm{Zu}$ dem genannten Termin fand ich zunächst eine auffallende Minderzabl der letzteren in den Haartaschen und Talgdrüsen gegenüber dem Befunde unmittelbar nach der Einreibung. Aber noch mehr: die Mehrzahl der noch vorhandenen Quecksilbertheilchen liess jene bereits beschriebene Umwandinng in eine Oxydulverbindung auf das Deutlichste an der trübschwarzen Beschaffenheit und eckigen Contour erkennen. Zugleich fiel auf, dass ein Theil der Talgdrüsen ausgesprochene Vergrösserung und fettig-körnigen Zerfall auch der peripherischen Zellen aufwies. Des Ferneren constatirte ich in den Haartaschen einer Leiche nach 3 maliger Inunction 4, 2 und 1 Tag vor dem Tode die Gegenwart oxydirter Metallkügelcben in gleicher Weise. Berücksichtigen wir neben diesen Befunden den bereits erwähnten Lewald'schen Nachweis der Löslichwerdung des regulinischen Metalls, wie des fettsauren Oxydes und Oxydules bei Contact mit buttersauren Verbindungen, so werden wir nicht im Zweifel darüber sein können, dass die in den Haartaschen und Talgdrüsen resp. deren Gängen deponirten Quecksilberkügelchen der grauen Salbe in Folge des dauernden Contactes mit dem (Eiweiss, Fett, flüchtige Fettsäuren, Kochsalz u. s. w. 
enthaltenden) Hauttalg durch die Phase der 0xydulbildung in eine lösliche wirksame Quecksilberverbindung übergeführt werden müssen, deren Resorption in die Säftemasse nichts mehr im Wege steht. Dieser Bildung von löslichem, also corrodirendem Quecksilber ist meines Erachtens auch die bei empfindlicher Haut nicht seltene Entwicklung des impetiginösen Eczem. mercurial., sowie die in loco applicationis voraneilende Involution recenter Syphiliden zuzuschreiben.

Wird somit das regulinische Quecksilber durch einfachen Contact mit Blut und Hauttalg in eine lösliche Verbindung übergeführt, ohne dass eine Bestreichung Seitens der Atmosphäre stattfindet, ist es ferner eine unbestrittene Thatsache, dass feinvertheiltes Quecksilber in Kochsalz-, Eiweiss-, Fettsäure-Lösungen etc. unter der Mitwirkung des Sauerstoffs der Luft ebenfalls eine lösliche Verbindung abgiebt, so bedarf es meines Erachtens kaum neuer Experimente, um das Schicksal des durch die Respiration aufgenommenen Metalldampfes, $z u$ eruiren. Schlägt sich äusserst fein vertheiltes aus dem Dampf condensirtes Quecksilber an den durch die verschiedensten Secrete feucht erhaltenen und grösstentheils von Luft bestrichenen Schleimhäuten der Mundhöhle und des Respirationsapparates nieder und gelangt es mit dem Speichel und der Nahrung in Magen und Darm ${ }^{1}$ ), so werden genügende Bedingungen zur 0xydation und somit Ueberführung in eine resorptionsfähige lösliche Verbindung gegeben sein, wie denn auch Rindfleisch durch den Nachweis fettig-körnigen Zerfalls der in Contact mit den Kügelchen gerathenen Zellen eine solche Ueberführung mehr als wahrscheinlich gemacht hat. Schliesse ich mich also bezüglich der Lehre von der Wirkung der Quecksilberdämpfe der von Herman $\mathbf{n}$ gegebenen Anschauung unbedingt an, so erübrigt noch, mit einigen Worten der Bedeutung des a u f der unverletzten Haut abgelagerten Quecksilbers d. i. der am Orte der Inunction liegen gebliebenen Reste der grauen Salbe

1) Die Contenta des Mundes (der schnelleren Oxydation des Quecksilbermetalles günstige Zersetzungsproducte) scheinen gleich der Aufnahme der dem Metalldampf längere Zeit zugänglich gewesenen Nahrung eine wesentliche Rolle zu spielen. Nach Kussmaul bleibt (a. a. 0. S. 222) der Quecksilber-Arbeiter, welcher den Mund rein hält, viel länger verschont, als der unreinliche und "Trinken und Essen in den Arbeitsräumen ist ausserst gefäbrlich und streng verboten ". 
und des durch Condensation des Dampfes an der feuchten Körperoberfläche deponirten Metalles zu gedenken. Auch hier muss schliesslich eine Sublimatbildung stattfinden, denn die 3 Momente: feine Vertheilung des Metalls, Benetzung mit dem Eiweiss, Fett, Fettsäuren, Kochsalz u. s. w. enthaltenden Hautsecret (Hauttalg und Schweiss) und Bestreichung von Luft sind auch hier gegeben. Eine andere Frage ist indess die nach der Resorption des gebildeten Sublimats. Sicher wird letztere stattfinden anch bei den mindesten Läsionen der Haut, wogegen eine Aufnahme von der unverletzten Haut nach dem heutigen Standpunkt der Lehre unwahrscheinlich ist.

Es bedarf schliesslich wohl kaum der Erwähnung, dass äberall da, wo die graue Salbe oder ihre Quecksilberdämpfe in Contact mit Haut- oder Schleimhauterosionen, Ulcerationen gelangen, neben der Einwanderung der Kügelchen in's klaffende Parenchym resp. Aufnahme in die Circulation auch die Bilaung einer löslichen 0xydationsstufe bei der Ablagerung des Metalles auf die Wund- und Geschwürsflächen und Resorption in dieser Form in Betracht kommt (cf. Rindfleisch a. a. 0. S. $313 \mathrm{ff}$.). -

Es wirkt also, um karz zu resumiren, die Inunctionskur mit frischer (von oxydirtem Quecksilber freie), grauer Salbe bei intacter Haut und Schleimhaut

1) dadurch, dass die Metallkügelchen am Orte der Application durch das Einreiben in die Haartaschen und Talgdrüsengänge gedrückt und unter dem Einfluss des Drüsensecretes in eine lösliche resorptionsfähige Verbindung übergeführt werden;

2) dadurch, dass der durch die Respiration aufgenommene Quecksilberdampf sich an den zugänglichen Schleimbäuten regulinisch verdichtet und bei der Ablagerung eine resorbirbare lösliche $0 x y-$ dationsstufe liefert.

Dagegen dringen weder metallische Bestandheile der grauen Salbe am Ort der Einreibung durch die unverletzte Haut, noch penetrirt der Metalldampf durch Schleimhaut oder Haut, um als solcher im Blute zu existiren.

$\mathrm{Zu}$ den genannten Wirkungsmomenten treten neue Factoren bei verletzter Haut und Schleimhaut; hier ist

3) zur Einwanderung von regulinischem Quecksilber in die Blutcirculation Gelegenheit gegeben, namentlich am Orte der Inunction bei Existenz blutender Hautstellen; sobald regulinisches 
Quecksilber in Contact mit dem lebenden Blute kommt, liefert es eine lösliche wirksame Verbindung;

4) von diesem mechanischen Eindringen der Metallkügelchen abgesehen, wird auch überall da, wo regulinisches Quecksilber (sei es direct, sei es durch Condensation der Dämpfe) sich auf verletzter Hant oder Schleimhaut (Wanden, Geschwüren etc.) ablagert durch einfacbe Contactwirkung eine lösliche resorbirbare Verbindung gebildet. Aus demselben Grunde wird

5) auch der auf der Körperoberfläche regulinisch niedergeschlagene Dampf wirksam, sobald seine durch die Hautsecrete löslich gewordenen Oxydationsproducte mit der Epidermis beraubten Hautbezirken in Contact kommen. -

Ueber die dem einzelnen Modus zukommende quantitative Aufnahme wage ich keine bestimmte Ansicht, halte überbaupt einen derartigen Nachweis aus Anlass der Misslichkeit quantitativer Quecksilberbestimmungen in den Se- und Excreten und der geradezu unmöglichen Fixirung der Zeitgrenze, bis zn welcher das aus den einzelnen Aufnahmequellen resultirende lösliche Quecksilber ausgefürt wird (Intermittenz der Ausscheidungen!) für undurchführbar, ganz abgesehen von den kaum zu überwindenden Schwierigkeiten der Trennung und des Ausscblusses der verschiedenen Resorptionswege. Immerhin glaube ich der mindesten Aufnahme, mithin der geringsten practischen Bedeutung des regulinisch in die Circulation gelangten Quecksilbers das Wort reden zu müssen, da die Bedingungen dieser Aufnahme (blutende Erosionen etc.) einmal nicht zur Regel zählen, des Ferneren bei den Injectionen in den Kreislauf der Thiere trotz ausserordentlich gesteigerter Oberflächenwirkung und bis wochenlangen Contactes nur geringste Mengen einer wirksamen Verbindung nachgewiesen werden konnten, endlich, weil der subcutanen Einverleibung von Metallemulsionen, wie ich gezeigt habe, eine äusserst träge Wirkung eigen jst $^{1}$ ) und diese Methode nie mit Intoxicationserscheinungen einhergeht. Dass aus dem einfachen Con-

1) Wie ich im 1. Nacbtrag zu der bereits erwähnten Abhandlung im 24. Bande des d. Arch. f. klin. Med. dargethan, babe ich subcutan von mir eingespritztes laufendes Quecksilber noch $1 \frac{1}{2} \mathrm{Jahre}$ nach der Injection in Form stecknadelbopfgrosser Kügelchen wíedergefunden. Hier war es zu narbigem Abschluss des Unterbautraumes gekommen, der die Locomotion des Metalles verbindert. Letzteres hatte als chemisch reizender Fremdkörper jabrelang bestehende clcerationen bedingt. 
tact des regulinischen Quecksilbers mit selbst kleinen Ulcerationsflächen (Aufnahme löslich gewordener Producte) bei Ausschluss von Einreibungen und der Aufnahme von Dämpfen beträchtliche Wirkungen resultiren können, ist durch zahlreiche Beobachtungen bekannt. Eines der bezeichnendsten Beispiele habe ich selbst jüngst auf meiner Abtheilung beobachtet: die Application kleiner Mengen ziemlich frischer grauer Salbe auf einen geringfügigen Schanker an der einen grossen Labie in Form eines einfachen Verbandes hatte schnell zu hochgradiger mercurieller Stomatitis geführt. Die wichtige Rolle, welche die - an und für sich niemals wirksamen Metalldämpfe durch Vermittelung der Aufnahme des Medicaments in wirksamer Form bei der Inunctionskur spielen, ist sattsam bekannt (Kirchgässer u. v. A.). Sie als ausschliessliche Quelle der Wirkung der grauen Salbe anzusprechen, verbietet schon das von uns erörterte Verhalten des eingeriebenen metalliscben Quecksilbers.

Jedenfalls sieht man, dass der Wirkung der grauen Salbe ein ganz eigenartig complicirter, Seitens der übrigen Mercurialisationen schwerlich erreichbarer Gharakter zukommt, und warum gerade bei der Inunctionskur im Organismus das Medicament in einer Weise deponirt wird, dass er von dem Vorrath längere Zeit zehren kann. Gerade bezüglich der Remanenz des Quecksilbers in Körper lehrt eine unbefangene Durchsicht der Literatur, dass die nachträgliche Ausscheidung ganz besonders bei der Inunctionskur beobachtet wird, wie denn auch aus den neuesten Untersuchungen von Vajda und Paschkis ${ }^{1}$ ) der Schlusssatz resultirt, dass , die Einreibung von grauer Salbe der chronischen Natur des Syphilisprozesses noch am meisten angepasst erscheint, $d$. i., wo es sich um eine anhaltende Wirkung handelt, noch am meisten taugt".

Eine eingehendere Kritik der practischen Bedeutung der Inunctionskur mit ihren mannichfachen Inconvenienzen und Vorzügen liegt ausserhalb des Planes dieser Arbeit. -

Die Herren Hofräthe Proff. Müller und Nothnagel haben mir für meine Untersuchungen freundlichst das erforderliche Leichenmaterial, sowie die nöthigen Laboratoriumsrequisiten zur Verfügung gestellt. Es ist mir eine liebe Pflicht, beiden Herren an dieser Stelle meinen herzlichsten Dank auszusprechen.

1) Ueber den Einfluss des Quecksilbers auf den Syphilisprozess. Wien 1880. S. 295. 